Dynamic investment strategies of hedge funds

Kristien Smedts and Jan Smedts

DEPARTMENT OF ACCOUNTANCY, FINANCE AND INSURANCE (AFI) 


\title{
Dynamic Investment Strategies of Hedge Funds
}

\author{
Kristien Smedts $^{\mathrm{a} *}$ and Jan Smedts ${ }^{\mathrm{b}}$ \\ ${ }^{a}$ AFI, Catholic University of Leuven \\ ${ }^{\mathrm{b}} \mathrm{CFA}$, Dexia Bank
}

\begin{abstract}
In this paper we study the investment dynamics employed by hedge fund managers. Using daily data for nine investable hedge fund strategies, we use a rolling-over regression technique, which allows us to capture the time-variability present in the different strategies of hedge fund managers. The results indicate that the inclusion of time-variability is important as the risk exposures change significantly over time. Our results show no evidence of traditional alpha out-performance within a multifactor framework. Given this inability to generate consistent alpha returns, we also analyze the performance data relative to the factor specific beta risk. To this end, we replicate static hedge fund returns and compare them to the actual hedge fund returns. We conclude that most hedge fund returns beat the replicated static trading strategy. This suggests that particular hedge funds add alpha return through the skill of timing alternative beta risk.
\end{abstract}

Keywords: Hedge funds, multifactor model, abnormal returns

JEL: G12, G29

\footnotetext{
* Address of correspondence: AFI, Catholic University of Leuven, Naamsestraat 69, 3000 Leuven, email Kristien.Smedts@econ.kuleuven.be, Tel: +32(0)16326739, Fax: +32(0)16326796. We thank the editor of the Financial Analyst journal, an anonymous referee and Stan Beckers for insightful comments and suggestions.
} 


\section{Introduction}

Hedge funds are expected to gain further ground versus traditional long-only funds and to become mainstream investment vehicles in the years to come. Much of their prominence has been linked to their capacity to generate active -or alpha returnsversus the predominantly beta inspired returns of their traditional colleagues. However, it seems that some of the mystique surrounding the performance wonders has been due to the inability of the industry to discern true alpha generation from exposure to alternative betas. Although exposure to the latter can be beneficial both in terms of return potential as well as from the viewpoint of diversification, it could be that investors can gain more cost effective exposure through other ways. Traditionally, performance evaluation for these skill-based strategies has been problematic, mainly driven by the lack of consistent performance data. For a review of the different difficulties, we refer to Ineichen (2002).

Separating beta risk exposure from alpha return is a hazardous exercise given the wide range of instruments and strategies employed by hedge funds. Hedge fund managers switch between asset classes, hold long as well as short positions, use leverage and use derivatives resulting in highly non-linear payoff structures. Hence, a multifactor model is the most obvious method to estimate hedge fund returns. A number of previous studies analyzed a variety of risk exposures of hedge funds. Among others, Fung and Hsieh (2002) adopt an asset-class multifactor model in the spirit of the style analysis of Sharpe (1992). Edwards and Caglayan (2001), on the other hand, employ the Fama-French style risk factors.

A further property of hedge funds is that they have a tendency to change risk exposures more often than traditional funds. Given the few investment restrictions and regulations, hedge fund managers are able to shift their exposures rapidly in response to a change in the risk-return tradeoff of the underlying investment opportunities. To enhance the understanding and assessment of this risk-return tradeoff in hedge funds, we analyze the dynamics of hedge funds' risk exposures through time. The importance of such time-varying risk exposures is also studied in Alexander and Dimitriu (2005), who use a regime switching model for hedge fund returns. We employ a multifactor model and estimate the risk exposures for fixed rolling-over windows. This analysis captures the time-variation in most investment strategies. Moreover, it also reveals any hidden risk exposures. A similar analysis has been conducted by McGuire et al. 
(2005) who employ a rolling-over estimation of Sharpe's style analysis on monthly hedge fund returns. In contrast to their study, we use daily investable hedge fund return data. Given the possibility of frequent changes in the investment approach, high frequency data should better capture this time-variation and non-linearity. Moreover, we can expect that the investability of the daily hedge fund indices introduces a particular (liquidity-induced) risk-return tradeoff in the data that is not present in the risk return profile of monthly data.

In a second step, we analyze to what extent we can replicate (out-of-sample) the hedge fund return series. As noted by Asness (2004a) hedge fund strategies have a tendency to move over time from alpha to systematic beta trading strategies. By exploiting arbitrage opportunities, the market becomes more efficient and alpha returns quickly disappear. Moreover, once a strategy becomes common and widespread, its corresponding return is more likely a beta return and thus a reward for taking up this common systematic risk. In a competitive market, such beta return can survive as a risk premium, while true alpha disappears over time. ${ }^{1}$ This is exactly what the current hedge fund business is experiencing. Alpha strategies that become mainstream, such as convertible arbitrage, no longer generate sufficient arbitrage profits so managers tend to take more directional alternative beta exposure. However, this does not imply that there is no more scope for out-performance. A second source of alpha return, namely the timing of (alternative) beta exposure, could still be realized. This could be one of the strengths of hedge funds. While the hedge funds' beta returns are similar concepts as traditional beta returns, their structure is much more complex, and their strategies demand expert knowledge and skills. Successful market timing of alternative betas can, as such, be interpreted as another important source of alpha return. Furthermore, unconstrained hedge fund structures have the potential to produce better outcomes than the more constrained traditional formats (see Beckers and Smedts (2004)). To test whether successful market timing is present in hedge fund returns, we re-estimate the multifactor model assuming that the estimated coefficients correspond to portfolio weights. This analysis allows us to get a better understanding of the (predictive) success of a particular hedge fund strategy. In the current context of daily investable indices, this analysis is particularly interesting.

\footnotetext{
${ }^{1}$ In equilibrium, beta that is rewarded for with a positive expected return corresponds to risk for which investors are willing to pay others to bear this risk. Also Jaeger and Wagner (2005) argue that the main component of hedge fund returns corresponds to risk premia rather than arbitrage opportunities.
} 
A hedge fund strategy that outperforms in an environment where daily liquidity is to be maintained, is clearly a very attractive investment. We find that several of the strategies indeed outperform the (static) replicated strategies. This is interpreted as evidence in favour of successful market timing by hedge fund managers.

The paper is organized as follows. In Section 2 we describe the hedge fund data used in the paper, and we briefly elaborate on potential biases. Section 3 explains the methodology of the asset-based multifactor model. Section 4 reports the estimation results of the factor model and presents the replication results. Finally Section 5 concludes.

\section{Hedge Fund Indices}

The hedge fund data used in the current paper are daily investable HFR indices. These HFR fund returns are actual ('live') and net of all fees and expenses. HFR reports daily hedge fund returns since 1/4/2003 for indices of 8 single strategies: convertible arbitrage (CA), distressed securities (DS), equity hedge (EH), equity market neutral (EMN), event-driven (ED), macro (M), merger arbitrage (MA) and relative value arbitrage (RVA). They also report a global hedge fund index (GL). These HFR daily indices are fully investable by means of trackers, designed to mimic the performance and features of each of these indices.

The objective of HFR is to create investable hedge fund returns that reflect the performance, composition and changing opportunities of the hedge fund universe. Of course, it is well known that both the index construction as well as the hedge fund strategies itself create flaws and biases into the return series. Interpreting these performance numbers and generalizing results should, therefore, always be done with caution. Specifically, the following biases should be kept in mind: a selection bias (both from the index constructor as well as from the hedge fund manager), a survivorship bias, backfilling and autocorrelation.

The hedge funds included in any index reflect only a small subset of the whole hedge fund universe. Every index provider uses its own selection and eligibility criteria and as shown by Fund and Hsieh (2004) the largest index providers have only few hedge funds in common. To be eligible for inclusion in the HFRX indices, a hedge fund needs to be open for investment, have at least USD 50 mio assets under management and have a track record of 24 months. Also, hedge funds included in the 
HFRX indices need to make available their trades and positions on a daily basis and offer daily pricing. Moreover, the final decision always lays with an index calculation committee that needs to approve the inclusion in the index. The choice of funds is thus rather non-transparent, and has likely a serious impact on performance numbers. For example, the design of an investable index and the selection criterion of open funds that report daily are intertwined: the indices represent daily liquidity providers. Most likely this comes at a serious cost, since both the available assets as well as the employed strategies are limited. These limitations probably show up in lower daily returns, but also in less autocorrelated returns, compared to monthly and closed hedge fund returns.

Of course, also the hedge funds themselves induce a self-selection bias. Hedge funds are private investment vehicles, and as such, are not obliged to disclose their activities. Not all hedge funds are willing to provide the necessary information to be included in a particular index. This biases the indices towards specific fund managers, and probably affects its risk-return profiles. This is for example true for the open-fund criterion. Very successful funds are often closed as they are not looking for additional investments. The capacity effect is, therefore, most likely to be an issue for top managers, resulting in a downward bias in the returns of open funds only. This point is also raised by Jaeger and Wagner (2005) who compare monthly HFR investable and non-investable indices and find a clear underperformance of most of the investable indices. The recent emergence of so called institutional hedge fund managers might exacerbate this self-selection bias. Some of these players started up their own hedge fund business merely to be present in the growing hedge fund market and to offer hedge fund products to their clients. The strategies and corresponding returns, however, do not resemble the top-players in the hedge fund business. Overall, we can expect that the selection bias of the index constructors and the self-selection bias of the hedge fund managers depress performance numbers of the indices compared to the overall hedge fund universe performance

Apart from the above mentioned (self-) selection bias, hedge fund return data also suffer from a survivorship and backfilling bias, both leading to an upward bias in the level of reported returns. For example, Malkiel and Saha (2005) estimate the survivorship and backfilling bias to 442 and 500 basis points, respectively. By using live data, rather than pro forma historical data both biases are minimized in our dataset. Of course, the biases are not fully eliminated. For example, even if the returns 
are not backfilled, it is true that only successful funds with a track record of 24 months are eligible for inclusion in the index. Unfortunately, we cannot exactly measure the particular size and seriousness of the different biases, but we can expect that the downward (self-) selection bias is partly offset by the upward backfilling and survivorship bias.

Finally, a last important element that can seriously distort hedge fund returns is autocorrelation, induced by the illiquidity of the hedge fund investments. Hedge fund managers are often invested in over-the-counter instruments and illiquid strategies, which are difficult to price. Asness et al. (2001) interpret this serial correlation as hedge fund portfolios not being marked to market in a timely way. Potential time lags and complexity in the pricing of these investments might induce a smoothing of returns, leading to less volatility (see Getmansky et al. (2004)). Hedge funds included in the HFRX indices need to make available their trades and positions on a daily basis. Moreover, to keep track of pricing accuracy and style purity, these investment holdings are independently re-priced. The independent pricing together with the underlying assets in which they can invest (maintaining a high liquidity) implies that these indices are likely less prone to price manipulations, leading to less autocorrelation compared to the serial correlation found in e.g. non-investable monthly indices. We therefore expect that the degree of autocorrelation is limited and is in line with the serial correlation that is also found in some other investment vehicles like small cap funds. To test for this hypothesis, we perform the Ljung-Box Q-test for serial correlation and find serial correlation in almost all of the indices. However, as expected, the degree of the serial correlation is smaller compared to the autocorrelation in monthly indices, found in previous studies (see Getmansky et al. (2004)). Similar results are also obtained by Boyson et al. (2006). Of course, to obtain an accurate view on the hedge fund risks, this autocorrelation should be accounted for. In line with Asness et al. (2001), we include a first-order autoregressive component in the estimation procedure.

Even if it is true that these biases do not disappear (or might even be aggravated) when focusing on investable indices, we think that the presence of these biases is not problematic in the context of the current analysis. While it is true that the hedge funds included in our study might exhibit biases and represent only a subset of the whole hedge fund universe, these hedge fund indices are traded instruments. An investor can buy trackers, specifically designed to replicate the full features and performance of 
each corresponding index and as such, they do reflect the actual risk-return profile that this investor is facing.

\section{Methodology}

To estimate excess returns for hedge fund indices, we use an eight-factor model:

$$
\mathrm{R}_{\mathrm{t}}-\mathrm{R}_{\mathrm{f}, \mathrm{t}}=\alpha+\sum_{\mathrm{k}=1}^{\mathrm{K}} \beta_{\mathrm{k}}\left(\mathrm{R}_{\mathrm{k}, \mathrm{t}}-\mathrm{R}_{\mathrm{f}, \mathrm{t}}\right)+\varepsilon_{\mathrm{t}}, \quad \forall \mathrm{t}=1, \ldots, \mathrm{T}
$$

where $R_{t}$ is the return of a particular hedge fund strategy, $R_{f, t}$ is the risk-free rate of return and $\mathrm{R}_{k, t}$ is the return on factor $\mathrm{k}$. The choice of asset-based factors is in line with previous studies (see, among others, Liang (1999)). In particular, we use the Russel 3000 index for the US equity market (Useq), the MSCI emerging markets index (Emeq), the Salomon Brothers government/corporate US bond index (Usb), the Salomon Brothers world ex-US government bond index (Wb), the Merill Lynch high yield global index (Hyb), the Goldman Sachs commodities index (Com), the Volatility Index (Vix) and an autoregressive component $(\operatorname{Ar}(1)){ }^{2}$ The risk-free rate is the US-Euro 1-week middle rate. All factor data are total returns in USD and are provided by Datastream.

We estimate time-varying exposures by a rolling-over OLS regression. We use a fixed window of 40 observations, corresponding to a buy-and-hold period of approximately 2 months. This should capture the highly dynamic investment strategy of hedge fund managers. As mentioned earlier, hedge fund returns are characterized by autocorrelation. Even if we include a first-order autoregressive component in the estimation, there is still serial correlation for some of the estimations. To correct for this, we use Newey-West standard errors. The Newey-West correction provides a way to calculate a consistent covariance matrix in the presence of serial correlation (and heteroskedasticity) and thus leads to correct statistical inference.

\footnotetext{
${ }^{2}$ In first instance we also included the MSCI world ex-US equity index. However, this factor is heavily correlated with the MSCI emerging markets equity index, and therefore we eliminated the former.
} 


\section{Estimation Results}

\subsection{Data Description}

Summary statistics for both the hedge fund returns and the factor returns are reported in Table 1. These summary statistics differ to some extent from the data reported in previous studies. Mean returns for the hedge fund portfolios are at the lower end on an historical basis and are significantly lower than returns reported previously (e.g. see Ranaldo and Favre (2003)). Although most return data presented are always coloured by the time frame chosen, this trend could indicate the lack of interesting (arbitrage) opportunities, mainly due to the large number of players and capital invested in some strategies. Also, these low average returns might be the result of the selection bias. Funds reporting on a daily basis are most likely invested in more liquid instruments and less complex transactions, depressing their returns. For example, the CA and RVA strategies likely miss the liquidity premium associated with complex deals.

Looking at the standard deviations, we see that the volatility of the hedge funds is in general much lower than the volatility of the factors. This highlights the classical benefits of diversification. The lower hedge fund returns have been accompanied with lower volatilities, mainly mirroring the trend in equity volatility since mid 2003 . This is also confirmed when analyzing the Sharpe ratios. Of course, in the context of hedge fund returns standard deviation as a measure of risk should be treated with caution. Hedge fund returns exhibit serial correlation, understating the true underlying volatilities. Also, the second moment is only a reasonable measure of risk when the underlying distribution is normal. Hedge fund return distributions, on the other hand, display skewness and kurtosis. This also also holds for the hedge fund returns in the current paper, as shown by the Jarque-Bera test. Also for most of the factor portfolios we have to reject normality, with the exception of the US equity portfolio and the world government bond portfolio. Finally, we also report the minimum and maximum returns. 


\section{Table 1: Summary Statistics}

The summary statistics are based on daily data from 1/4/2003 until 31/07/2006 and are expressed in annualized USD terms. We report the mean return, the standard deviation, the Sharpe ratio, the minimum and maximum return and finally the p-value of the JarqueBera normality test.

\begin{tabular}{lcccccc}
\hline & Mean & St. Dev. & $\begin{array}{c}\text { Sharpe } \\
\text { Ratio }\end{array}$ & Min & Max & J.B. \\
& & & & & \\
\hline CA & 0.005 & 0.034 & -0.580 & -2.318 & 2.026 & 0.00 \\
DS & 0.081 & 0.026 & 2.183 & -1.834 & 1.925 & 0.00 \\
EH & 0.056 & 0.050 & 0.624 & -2.842 & 3.767 & 0.00 \\
EMN & $5 \mathrm{E}-04$ & 0.028 & -0.882 & -1.540 & 2.025 & 0.00 \\
ED & 0.084 & 0.038 & 1.545 & -2.624 & 2.605 & 0.00 \\
M & 0.056 & 0.055 & 0.566 & -4.170 & 2.862 & 0.00 \\
MA & 0.048 & 0.028 & 0.833 & -2.162 & 1.787 & 0.00 \\
RVA & 0.037 & 0.028 & 0.418 & -2.365 & 4.065 & 0.00 \\
GL & 0.054 & 0.029 & 0.976 & -1.918 & 2.042 & 0.00 \\
Useq & 0.156 & 0.120 & 1.094 & -5.957 & 6.336 & 0.53 \\
Emeq & 0.345 & 0.151 & 2.115 & -11.840 & 10.454 & 0.00 \\
Usb & 0.031 & 0.042 & 0.147 & -2.865 & 2.442 & 0.00 \\
Wb & 0.064 & 0.092 & 0.427 & -4.546 & 4.721 & 0.51 \\
Hyb & 0.115 & 0.029 & 3.098 & -1.968 & 1.861 & 0.00 \\
Com & 0.257 & 0.234 & 0.992 & -11.613 & 16.889 & 0.01 \\
Vix & 0.145 & 0.828 & 0.145 & -64.772 & 77.139 & 0.00 \\
\hline
\end{tabular}

\subsection{Factor Model Estimation}

To get a clear understanding of the time-variation in the hedge funds' risk exposures, we first report the (constant) full sample results for the eight-factor model in Table 2. For three of the strategies, ED, EH and M, the hedge fund return dynamics are fitted well by the multifactor model. Also, for the global hedge fund index GL the factor model explains a large part of the variance. In terms of the risk exposures, the factor high yield bonds $(\mathrm{Hyb})$ is the most important one. Not only are its factor loadings largest, this factor is also often estimated significantly (at the $1 \%$ level). To some extent this does not come as a surprise given the overall performance of the credit markets over the period studied. Also the factors emerging markets (Emeq) and volatility (Vix) are very important. Its factors loadings are not as large as the Hybfactor, but eight and seven of the strategies, respectively, load significantly to these 
factors. Clearly, these results indicate that most hedge fund managers are hardly constrained and try, at times successfully, to grab a slice of the cake.

Our results for the CA strategy also seem to confirm the transition the strategy has made from a historical long volatility tilt towards a more credit driven strategy. A similar result holds for the strategy RVA. To the extent that the RVA style mimics $\mathrm{CA}$, we find similar signs of the factor exposures (apart from the Useq factor). The risk profile for ED seems, not surprisingly, to mirror the EH strategy, highlighting the significant equity exposure for both strategies. The inconclusive results for EMN might be due to the difficulties the strategy was encountering with the continued drop in overall equity volatility during our sample period. Commodity exposure is significant for $\mathrm{M}, \mathrm{ED}$ and $\mathrm{EH}$; whereas it is part of the natural opportunity set for macro-managers, it is likely through secondary exposure in the ED and EH strategy (e.g. through equity investments in the energy sector). Also worth noting is that 2 out of 3 strategies are short volatility proving that hedge funds have a tendency to operate as liquidity providers for financial markets. Finally, the autoregressive term is estimated significant for half of the strategies, and the inclusion of a first-order autoregressive term often removes the serial correlation that is otherwise present. This autoregressive coefficient is estimated positive in 3 cases. It is exactly this positive autoregressive component that could indicate stale pricing.

The estimates of the constant alpha returns (or so called Jensen's alpha or 'managerial skill') are also striking: Jensen's alpha is only in 1 case estimated positive, but not significant, while we find two strategies for which 'managerial skill' in fact significantly lowered hedge funds' excess returns. This indicates that a lot of market players are active and that alpha returns have largely been arbitraged away. ${ }^{3} \mathrm{~A}$ similar conclusion is also reached by Loeys and Fransolet (2004). They find that opportunities are fast disappearing in market segments were a lot of players are active, where the trading rules used are common knowledge, and where derivatives markets are deep. Finally, it is also interesting to note that the order of magnitude of the constant correlates well with the typical 1-2\% fixed management fee.

\footnotetext{
${ }^{3}$ Of course, given that the empirical analysis uses hedge fund indices, the alpha estimates reflect the average alpha within a certain style. Such alpha estimates are likely biased towards zero.
} 


\section{Table 2: Factor Model Estimation (full sample)}

The table reports the full sample eight-factor model estimates with HAC standard errors. ${ }^{*}$ indicates significance at the $5 \%$ level and ${ }^{* *}$ at the $1 \%$ level. We also report the model fit as the adjusted $\mathrm{R}^{2}$.

\begin{tabular}{llllllllll}
\hline & CA & DS & ED & EH & EMN & M & MA & RVA & GL \\
\hline Cte & $-2 \mathrm{E}-4$ & $1 \mathrm{E}-4$ & $-2 \mathrm{E}-5$ & $-2 \mathrm{E}-4^{* *}$ & $-1 \mathrm{E}-4$ & $-2 \mathrm{E}-4$ & $-1 \mathrm{E}-5$ & $-4 . \mathrm{E}-5$ & $-1 \mathrm{E}-4^{* *}$ \\
Useq & $-0.065^{* *}$ & -0.015 & $0.129^{* *}$ & $0.217^{* *}$ & $-0.041^{* *}$ & 0.003 & $0.060^{* *}$ & 0.023 & $0.101^{* *}$ \\
Emeq & 0.005 & $0.014^{*}$ & $0.072^{* *}$ & $0.110^{* *}$ & $0.034^{* *}$ & $0.159^{* *}$ & $0.015^{*}$ & $0.017^{*}$ & $0.080^{* *}$ \\
Usb & 0.015 & -0.034 & -0.031 & 0.022 & $0.061^{*}$ & 0.015 & -0.014 & 0.005 & 0.008 \\
Wb & -0.022 & $-0.027^{* *}$ & $-0.064^{* *}$ & -0.012 & 0.016 & $0.101^{* *}$ & -0.017 & -0.010 & -0.006 \\
Hyb & $0.268^{* *}$ & $0.281^{* *}$ & $0.313^{* *}$ & 0.023 & -0.037 & 0.028 & $0.113^{* *}$ & $0.175^{* *}$ & $0.119^{* *}$ \\
Com & -0.002 & -0.002 & $0.008^{*}$ & $0.015^{* *}$ & $-3 \mathrm{E}-04$ & $0.057^{* *}$ & 0.002 & -0.002 & $0.014^{* *}$ \\
Vix & 0.001 & $-0.003^{*}$ & $-0.008^{* *}$ & $-0.011^{* *}$ & $-0.008^{* *}$ & $0.005^{*}$ & $-0.005^{*}$ & 0.002 & $-0.005^{* *}$ \\
Ar(1) & $-0.115^{* *}$ & $0.095^{*}$ & -0.035 & $0.108^{* *}$ & 0.037 & 0.034 & -0.061 & $-0.175^{* *}$ & $0.067^{* *}$ \\
$\mathrm{R}^{2}$ & 0.093 & 0.113 & 0.597 & 0.748 & 0.061 & 0.357 & 0.190 & 0.070 & 0.706 \\
\hline
\end{tabular}

Unfortunately, for several of the hedge fund strategies the seven-factor model only explains a minor part of the return variability. This is captured in the small and often insignificant risk exposures as well as in the low coefficient of determination. This finding is in line with the interpretation that hedge funds are not traditional investments, and that they do not exhibit the same risk characteristics as e.g. mutual funds. For example, several studies point to the non-linear risks to which hedge funds are possibly exposed (see Huber and Kaiser (2004)).

However, the lack of explanatory power of the above multifactor model might also be explained by the underlying assumption of a fixed set of parameters. Given that hedge fund managers are known to follow dynamic trading strategies and tend to rebalance their portfolios frequently, the assumption of fixed parameters over a long horizon might not be suitable. Allowing for structural changes to capture the changing investment style is probably more appropriate. Ideally, one should analyze sample lengths corresponding to the specific investment horizon of the manager. For our analysis we arbitrarily set the sample length to 40 observations, corresponding to a buy-and-hold strategy for a period of approximately 2 months. ${ }^{4}$ The estimation results are reported in Table 3 below. Given the large number of estimations of the rolling-over analysis, we only report some descriptive statistics of the (time-series of)

\footnotetext{
${ }^{4}$ Of course, the choice of sample length is, ultimately, ad hoc, but we believe that a fixed investment horizon of two months is plausible.
} 
estimates. Table 3 displays the mean and the coefficient of variation (ratio of standard deviation to mean) of the different risk exposures, together with the percentage of significant estimates. ${ }^{5}$ The same summary statistics are reported for the adjusted $\mathrm{R}^{2}$.

Several important observations can be made. First, there are often large differences between the level of the full-sample estimates and the average of the rolling-over estimates. Clearly, both would be similar if there is no considerable time-variability. Given that this is not the case, assuming a fixed set of parameters is probably not appropriate. This is also confirmed when analyzing the risk exposures in Figure 1. This figure plots, for the strategy CA, the time-series of factor risk exposures as well as the constant full sample factor risk exposures. The figure clearly shows that the full-sample estimates hide highly dynamic factor exposures. Moreover, most of the risk exposures change signs several times, implying that the investment strategy consists of alternating short and long positions in the corresponding factors. Hence the long-horizon fixed parameters embrace a wide range of different short horizon exposures. This result is obtained for most of the hedge fund strategies. The same conclusion is supported when looking at the coefficients of variation (CV) in Table 3. The considerable CV's indicate a wide dispersion in the estimated risk exposures. These ratios are extremely large, indicating that for most strategies, the risk exposures are unstable over time. For a few risk strategies/exposures, we find a rather low dispersion, indicating a more stable exposure over time. For example, this is true for the exposure to Useq in the strategies ED, EH and GL. However, in general there is considerable time-variation and switching in the underlying investment strategies.

\footnotetext{
${ }^{5}$ The full results are available from the authors on request.
} 


\section{Table 3: Factor Model Estimation (fixed rolling-over windows)}

The table reports the eight-factor model estimates for the fixed rolling-over window of size 40 . We report the mean and the coefficient of variation (ratio of standard deviation to mean) of the estimates, along with the percentage of significant estimates at the $5 \%$ level (based on HAC standard errors). We also report the mean and the coefficient of variation of the adjusted $\mathrm{R}^{2}$.

\begin{tabular}{|c|c|c|c|c|c|c|}
\hline & \multicolumn{3}{|c|}{$\mathrm{CA}$} & \multicolumn{3}{|c|}{ DS } \\
\hline & Mean & $\mathrm{CV}$ & $\%$ Sign & Mean & $\mathrm{CV}$ & $\%$ Sign \\
\hline Cte & $-1 \mathrm{E}-4$ & -5.40 & 27 & $1 \mathrm{E}-4$ & 3.10 & 28 \\
\hline Useq & -0.065 & -1.17 & 26 & -0.004 & -14.15 & 13 \\
\hline Emeq & 0.003 & 12.16 & 10 & 0.009 & 5.97 & 34 \\
\hline Usb & 0.049 & 4.75 & 51 & -0.058 & -2.20 & 19 \\
\hline $\mathrm{Wb}$ & -0.016 & -5.77 & 13 & -0.035 & -1.85 & 26 \\
\hline Hyb & 0.149 & 2.11 & 31 & 0.282 & 0.93 & 48 \\
\hline Com & -0.005 & -6.67 & 23 & 0.002 & 11.02 & 21 \\
\hline Vix & 0.001 & 7.30 & 12 & -0.002 & -3.44 & 18 \\
\hline $\operatorname{Ar}(1)$ & -0.201 & -0.68 & 44 & 0.024 & 8.81 & 38 \\
\hline \multirow[t]{3}{*}{$\mathrm{R}^{2}$} & 0.165 & 0.87 & & 0.124 & 1.02 & \\
\hline & \multicolumn{3}{|c|}{ ED } & \multicolumn{3}{|c|}{$\mathrm{EH}$} \\
\hline & Mean & $\mathrm{CV}$ & \% Sign & Mean & $\mathrm{CV}$ & $\%$ Sign \\
\hline Cte & $4 \mathrm{E}-5$ & 8.56 & 29 & $-1 E-4$ & -2.48 & 30 \\
\hline Useq & 0.169 & 0.51 & 82 & 0.258 & 0.40 & 92 \\
\hline Emeq & 0.059 & 0.68 & 51 & 0.107 & 0.55 & 79 \\
\hline Usb & -0.067 & -1.66 & 21 & -0.029 & -3.69 & 15 \\
\hline $\mathrm{Wb}$ & -0.070 & -0.86 & 45 & -0.031 & -1.77 & 18 \\
\hline Hyb & 0.339 & 0.63 & 58 & 0.113 & 1.88 & 24 \\
\hline Com & 0.006 & 3.89 & 26 & 0.008 & 2.23 & 25 \\
\hline Vix & -0.001 & -6.08 & 24 & -0.004 & -2.63 & 17 \\
\hline $\operatorname{Ar}(1)$ & -0.074 & -2.48 & 26 & 0.053 & 1.80 & 18 \\
\hline $\mathrm{R}^{2}$ & 0.617 & 0.26 & & 0.765 & 0.14 & \\
\hline
\end{tabular}


Table 3 - continued

\begin{tabular}{|c|c|c|c|c|c|c|}
\hline & \multicolumn{3}{|c|}{ EMN } & \multicolumn{3}{|c|}{ M } \\
\hline & Mean & $\mathrm{CV}$ & $\%$ Sign & Mean & $\mathrm{CV}$ & $\%$ Sign \\
\hline Cte & $-4 \mathrm{E}-5$ & -6.75 & 12 & $-3 \mathrm{E}-4$ & -1.71 & 28 \\
\hline Useq & 0.003 & 30.53 & 25 & -0.014 & -7.15 & 16 \\
\hline Emeq & 0.011 & 4.83 & 30 & 0.162 & 0.58 & 71 \\
\hline Usb & 0.003 & 50.85 & 22 & -0.007 & -55.82 & 42 \\
\hline $\mathrm{Wb}$ & 0.022 & 3.56 & 25 & 0.080 & 2.33 & 44 \\
\hline Hyb & -0.008 & -37.35 & 22 & 0.089 & 4.42 & 21 \\
\hline Com & -0.002 & -15.20 & 32 & 0.050 & 0.97 & 48 \\
\hline Vix & $-4 \mathrm{E}-04$ & 25.62 & 26 & 0.001 & 9.81 & 19 \\
\hline $\operatorname{Ar}(1)$ & 0.008 & 23.32 & 21 & -0.011 & -10.66 & 14 \\
\hline $\mathrm{R}^{2}$ & 0.174 & 1.00 & & 0.521 & 0.42 & \\
\hline \multicolumn{4}{|c|}{ MA } & \multicolumn{3}{|c|}{ RVA } \\
\hline & Mean & $\mathrm{CV}$ & $\%$ Sign & Mean & $\mathrm{CV}$ & $\%$ Sign \\
\hline Cte & $4 \mathrm{E}-5$ & 9.56 & 33 & $1 \mathrm{E}-5$ & 23.63 & 15 \\
\hline Useq & 0.082 & 1.13 & 53 & 0.034 & 2.71 & 34 \\
\hline Emeq & 0.017 & 2.49 & 25 & 0.012 & 3.24 & 18 \\
\hline Usb & -0.038 & -3.72 & 23 & -0.034 & -4.89 & 35 \\
\hline $\mathrm{Wb}$ & -0.022 & -2.64 & 20 & -0.013 & -6.61 & 31 \\
\hline Hyb & 0.135 & 2.25 & 21 & 0.173 & 1.55 & 37 \\
\hline Com & -0.002 & -13.20 & 27 & -0.004 & -7.24 & 20 \\
\hline Vix & -0.002 & -5.17 & 25 & 0.001 & 9.77 & 24 \\
\hline $\operatorname{Ar}(1)$ & -0.145 & -1.24 & 43 & -0.126 & -1.59 & 30 \\
\hline $\mathrm{R}^{2}$ & 0.283 & 0.62 & & 0.195 & 0.76 & \\
\hline \multicolumn{7}{|c|}{ GL } \\
\hline & Mean & $\mathrm{CV}$ & \% Sign. & & & \\
\hline Cte & $-1 \mathrm{E}-4$ & -2.48 & 31 & & & \\
\hline Useq & 0.125 & 0.45 & 87 & & & \\
\hline Emeq & 0.076 & 0.46 & 86 & & & \\
\hline Usb & -0.027 & -4.05 & 33 & & & \\
\hline $\mathrm{Wb}$ & -0.020 & -2.50 & 33 & & & \\
\hline Hyb & 0.173 & 0.97 & 44 & & & \\
\hline Com & 0.010 & 1.42 & 42 & & & \\
\hline Vix & -0.001 & -4.40 & 20 & & & \\
\hline $\operatorname{Ar}(1)$ & 0.001 & 61.83 & 11 & & & \\
\hline $\mathrm{R}^{2}$ & 0.725 & 0.20 & & & & \\
\hline
\end{tabular}


The CV's also allow us to compare the variation among the different exposures and strategies. This comparison shows us that the variation is largest for the smaller (in absolute value) risk exposures. Hence, it does not hold that the most widely dispersed factors determine the dynamics of the hedge fund returns. Therefore, to get a clear understanding of the factors driving the time-variation in the returns, not only the degree of dispersion is important, but also the level of the associated risk exposure. For example, the factors Vix and Com are in most cases quite dispersed, but
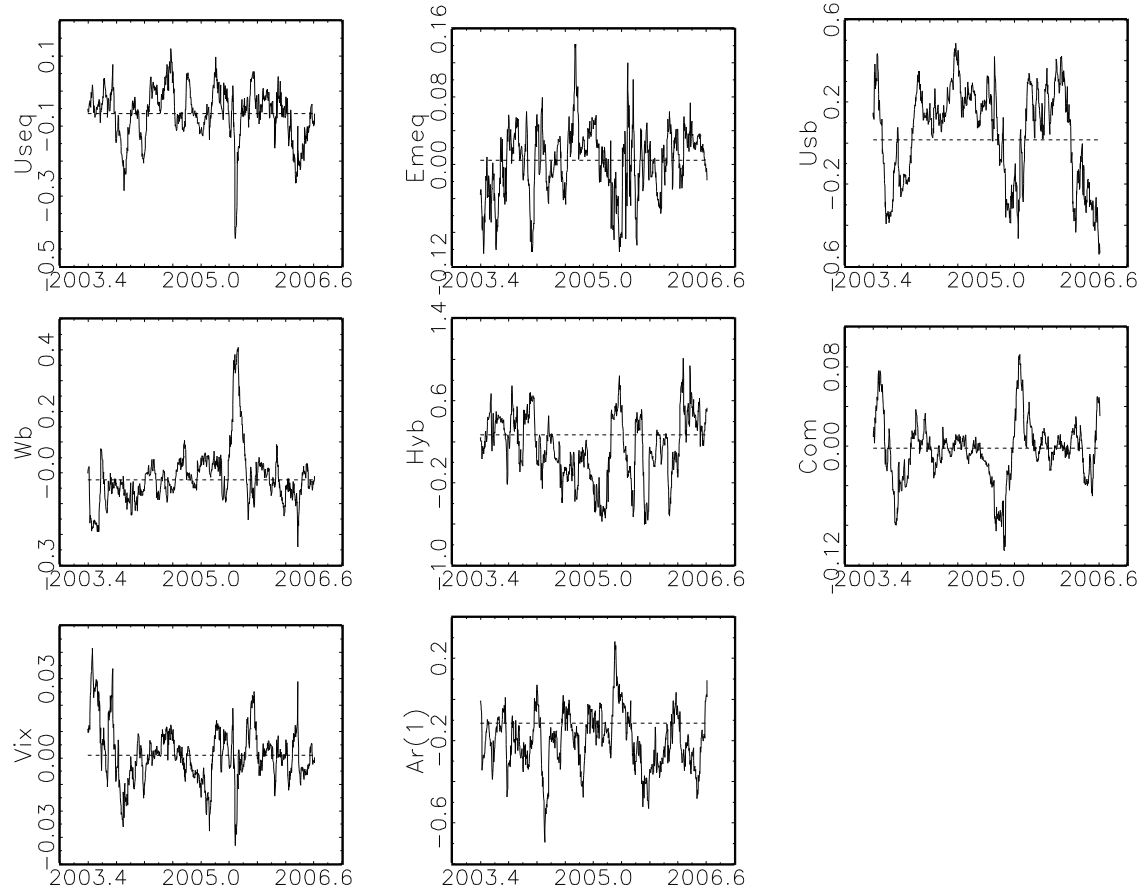

The graphs show the risk exposures of the rolling-over regressions (full line) and of the full sample regressions (dotted line), implied by the eight-factor model (equation (1)).

\section{Figure 1: Time-variation of the rolling-over exposures of the CA strategy}

their associated (mean) level of factor loading is rather small. The factor Hyb, on the contrary, only has an intermediate degree of dispersion, but the size of the (mean) risk exposure is relatively large. Thus, the time-variation in the hedge fund returns might largely be driven by such a factor. This is confirmed when analyzing the box-plot in Figure 2. This figure shows the shape of the distribution, its central value (median), the variability $\left(25^{\text {th }}\right.$ and $75^{\text {th }}$ percentile) and the minimum and maximum values for 
the strategy CA. We clearly see that the risk exposures change in our sample and that the risk factors Usb and Hyb are most important in absolute terms. ${ }^{6}$

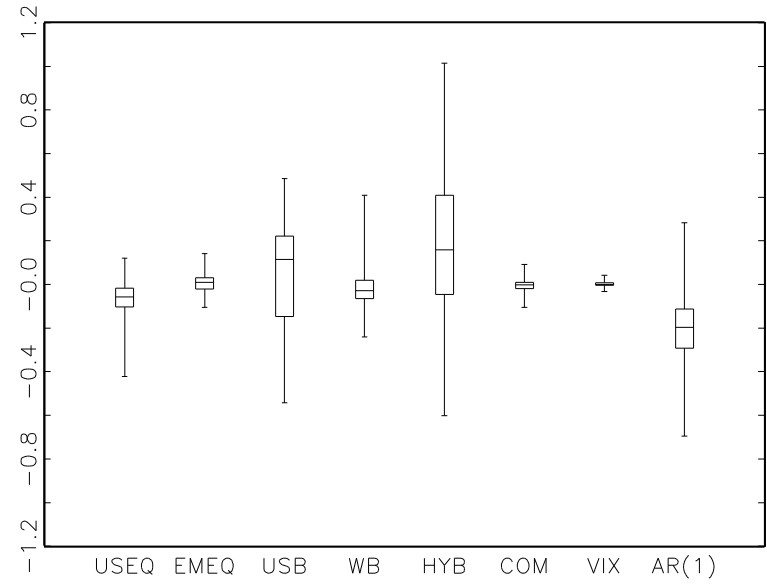

The graph shows the distribution of the risk exposures of the rolling-over regression of the CA strategy. This box-plot shows the median, the variability $\left(25^{\text {th }}\right.$ and $75^{\text {th }}$ percentile) and the minimum and maximum values for the CA.

\section{Figure 2: Importance of the rolling-over exposures of the CA strategy}

Apart from the economic importance of allowing for the time-varying risk exposures, we also analyze the statistical significance. Some risk exposure estimates turn out to be significant in the small rolling-over samples, while they are statistically insignificant for the full sample. Remarkable is, for example, the case of the CA strategy. For the full sample regression, we cannot reject that the factor US bonds is significantly different from zero. However, when considering the fixed rolling-over windows, we find that the same factor is estimated significantly for half of the rollingover regressions. Interestingly, the latter is more in line with what we expect. While the full sample results lead us to conclude that the CA strategy is not exposed to US bond risk, the short horizon estimates show that this bond factor is an important risk component. A similar interesting result is obtained for the strategy EMN. Relying on the full sample result, we find that equity market neutral managers are significantly exposed to US equity risk and emerging markets equity risk. Given the assumed strategy, this should come as a surprise. Luckily, the rolling-over estimations clarify this result: the factors Useq and Emeq turn out to be significant in, respectively, just less than one fourth and one third of the times. Importantly, the level of the Useq and

\footnotetext{
${ }^{6}$ See Appendix A for the box-plots of the other strategies.
} 
Emeq exposures also turn out to be much lower compared to the level obtained in the full sample. Noteworthy are also the results for the factor Usb. While the full sample results indicate that the EMN strategy is the only strategy that is (significantly) exposed to US bond market risk, the rolling-over estimations show that several of the strategies have a significant exposure to this factor. We already noted the significant exposure of the CA strategy to Usb (in 50\% of the cases), but also the strategies $\mathrm{M}$ and RVA are often significantly exposed to this bond factor (up to $42 \%$ of the cases for the $\mathrm{M}$ strategy). A similar conclusion is reached for the exposure to the commodities market. The different hedge fund strategies seem to take up more commodities risk than it appears at first sight. Finally, note that the exposure to the volatility factor is, in general, weakened when considering the rolling-over estimations.

An important reason for such different results of the full-sample versus the rollingover regressions is that hedge fund managers are allowed to hold both long and short positions. When a fund manager switches between long and short positions in a certain risk factor over time, its average exposure might turn out to be zero over a long horizon. The rolling-over strategy, on the other hand, does capture the intermediate investment positions when the fund is either long or short. This is also translated in the sign of the risk exposures that we estimate. For some of the risk factors we find that the majority of rolling-over regressions and the full-sample regressions produce different signs of the exposures. While a single large (long or short) exposure can heavily influence the full-sample results, this influence is only limited in time for the rolling-over regressions. The latter produces, of course, more reliable sign estimates.

The importance of allowing for time-variation also follows from the model fit. For all of the trading strategies, the average adjusted $R^{2}$ is higher than the adjusted $R^{2}$ of the full sample. For some of the strategies the increase in explanatory power is substantial, e.g. for CA, EMN, M, MA and RVA. The strategy DS is the strategy for which we observe almost no improvement in the model fit. A closer look at the estimation results also shows that this is a strategy where the averages of the rollingover estimates are similar to the full sample estimates. Time-variation seems to be less important for DS managers. For the other strategies, however, this confirms our finding that it is important to capture the dynamics of the investment strategies. This can also be seen in Figure 3 that plots the adjusted $R^{2}$ of the rolling-over regressions 
and of the full sample regressions. These graphs not only show that the rolling-over $\mathrm{R}^{2}$ are, in general, higher compared to the full sample $\mathrm{R}^{2}$, it also points to the (still) large variation in the explanatory power of the factor model. For certain periods, the eight-factor model seems to capture most of the variation in the hedge fund return series. For other periods, the model only offers a poor explanatory framework. This indicates that still some other risk factors are at work.

Overall, these results indicate that the long horizon estimations should be interpreted with caution. Considering shorter horizons disentangles the true underlying risk exposures of hedge funds and confirms that hedge fund managers employ dynamic trading strategies, allowing their risk exposures to vary significantly over time. Moreover, it also shows that hedge funds dynamically switch between asset classes. While some factors have an overall importance, the significance of other risk factors seems to be more limited over shorter horizons. This could point to the opportunity driven focus of hedge fund managers.
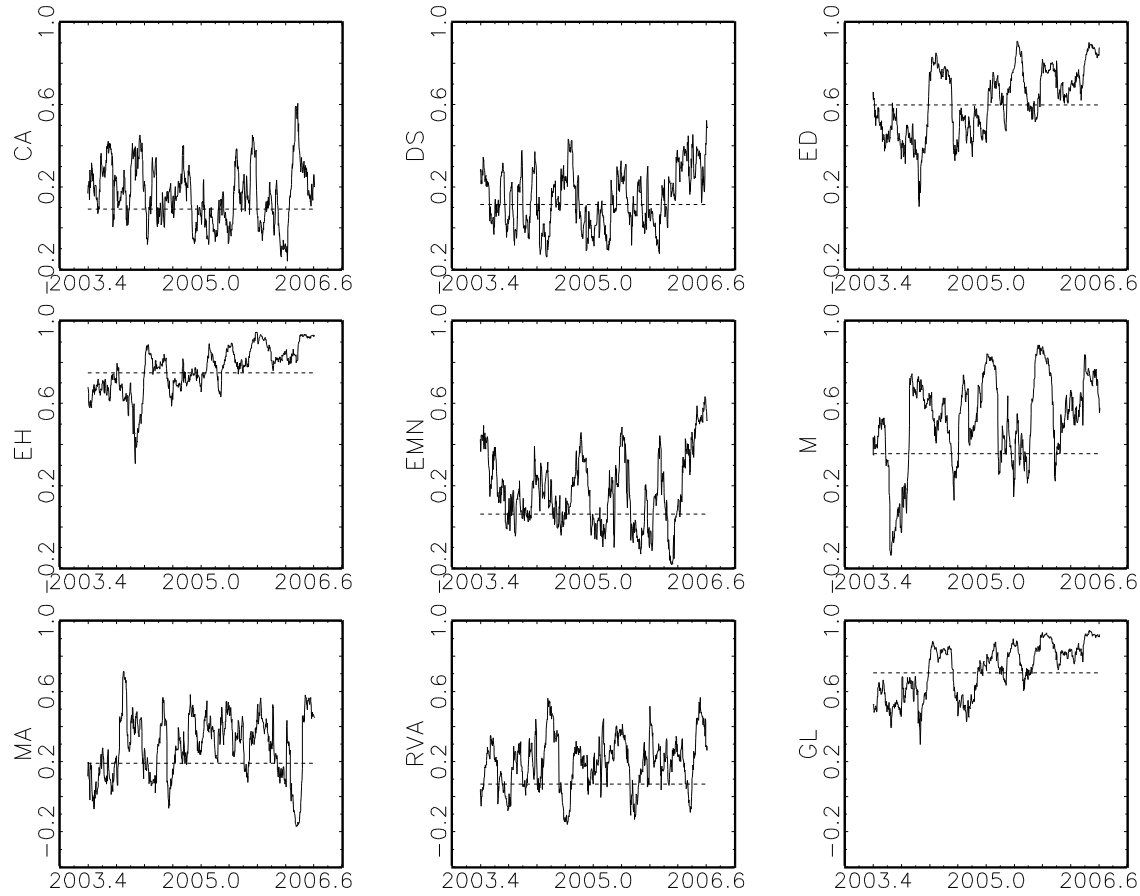

The graphs show the adjusted $\mathrm{R}^{2}$ of the rolling-over regressions (full line) and of the full sample regressions (dotted line), implied by the seven-factor model (equation (1)).

\section{Figure 3: Time-variation of adjusted $\mathbf{R}^{2}$ of the rolling-over estimations}

Finally, when considering the alpha estimates the conclusion remains largely the same as before: on average, hedge fund managers are not able to generate consistent 
alpha returns over and above their alternative risk exposures. Even worse, when the estimate of the constant alpha is significant, it is most of the time with a negative sign. This clearly shows that the most obvious pricing inefficiencies in the traditional markets have disappeared due to competition. As noted by Asness (2004b) this conclusion does not come as a surprise, since true skill is a zero-sum game. Hedge funds push markets into a (more) efficient equilibrium. A similar conclusion is reached by Géhin and Vaissié (2005) who note that excess returns through dynamic betas depend primarily on the skill of the hedge fund manager to time the factors with success, while arbitrage alpha returns are constrained by the amount of market opportunities. Hence the need for the hedge fund industry to be innovative (e.g. the explosion of the credit derivatives market) and remain quick footed to exploit new opportunities. Amenc et al. (2004) reach a similar conclusion. They note that the hedge fund industry should focus more on its normal beta benefits as opposed to its abnormal alpha returns, for alpha returns are hard to measure and arguable, and cannot be sustained in equilibrium. Also it should serve as a reminder that the source of beta return can change dramatically and that the appropriate risk framework should be employed when analyzing hedge fund returns. Only a clear understanding of the inherent risks allows investors to profit from the return and diversification potential of hedge funds.

\subsection{Replication of the dynamic strategies}

As argued earlier, market timing is a second potential source of alpha return. In this section, we analyze whether the dynamic beta exposures translate into such alpha return. If hedge fund managers are skilled to quickly rebalance their portfolio positions to profit from new investment opportunities, a passive (lagged) replication strategy underperforms their dynamic trading strategies. If this is the case, hedge funds add value ('alpha') through timing beta. A similar mimicking strategy has been performed by Jaeger and Wagner (2005) using monthly data. The main difference is that we replicate the hedge fund series by re-estimating the eight-factor rolling-over regressions as a Sharpe style model. ${ }^{7}$ This implies an additional assumption that the

\footnotetext{
${ }^{7}$ This is a first reason for the different results between the Jaeger and Wagner (2005) paper and the current paper. They find outperformance of monthly mimicking strategies based on a factor estimation. However, since they do not impose the exposures to correspond to portfolio weights, their analysis is
} 
risk exposures add up to one, giving them an interpretation of portfolio weights. Imposing this restriction worsens our estimation and fit of the model, but it yields a feasible replication strategy. Our methodology is as follows: we estimate the style model for the first window. Based on the estimated portfolio weights, we replicate the return for the (fixed-window +1 )th observation to the (fixed-window +40 )th observation, thus assuming a buy-and-hold horizon of 40 periods. ${ }^{8}$ This procedure is repeated for each consecutive buy-and-hold horizon of 40 periods and results in a series of out-of-sample replicated returns. These replicated returns are then compared to the actual returns of the hedge funds. This approach allows us to focus on the timing effect of the hedge fund managers, as the replicated strategies are always based on the 'old' factor exposures. If the hedge fund manager can successfully time his exposures, the actual strategy outperforms the replicated (lagged) hedge fund strategy. We interpret this timing effect as a source of alpha return. The results of this analysis can be found in Figure 4. This figure plots, for each strategy, the total return index based on its actual return and an index based on the replicated return. We see that for most of the hedge fund strategies in our study, the hedge fund index is superior to our passively replicated index. This is most pronounced for the strategy EMN. EMN managers are clearly able to time the risk factors, changing their investment strategy in a successful way. Instead of generating traditional alpha returns, they (perfectly) time alternative beta risk. The employed dynamics are, thus, important in generating superior returns. We can therefore conclude that this market timing translates into alpha returns. For most other strategies, we find that outperformance is smaller, but still positive. Also noteworthy is the variation over time in the success of a strategy to outperform a passive replication. For example, the CA strategy is able to outperform during most of the sample period, but seems to run into difficulties in the most recent period. The CA managers were clearly 'mis'-timing the market and a passive strategy would have yielded better returns. Looking more in detail at the particular situation, this represents most likely the impact of the General Motors debacle, pushing some CA hedge funds in serious financial problems and increasing the fear of a collapse of

more a rolling over out-of sample fit. We obtain similar results to Jaeger and Wagner (2005), when we do not impose a constraint on the coefficients.

${ }^{8}$ This implies that the portfolio is rebalanced approximately every 2 months, keeping the costs incurred from transacting reasonable. 
some financial institutions. ${ }^{9}$ A similar reasoning might hold for the DS managers. Like CA managers, DS managers are also long in bonds and short in equity, and likewise we see a tightening of their outperformance in the last months.
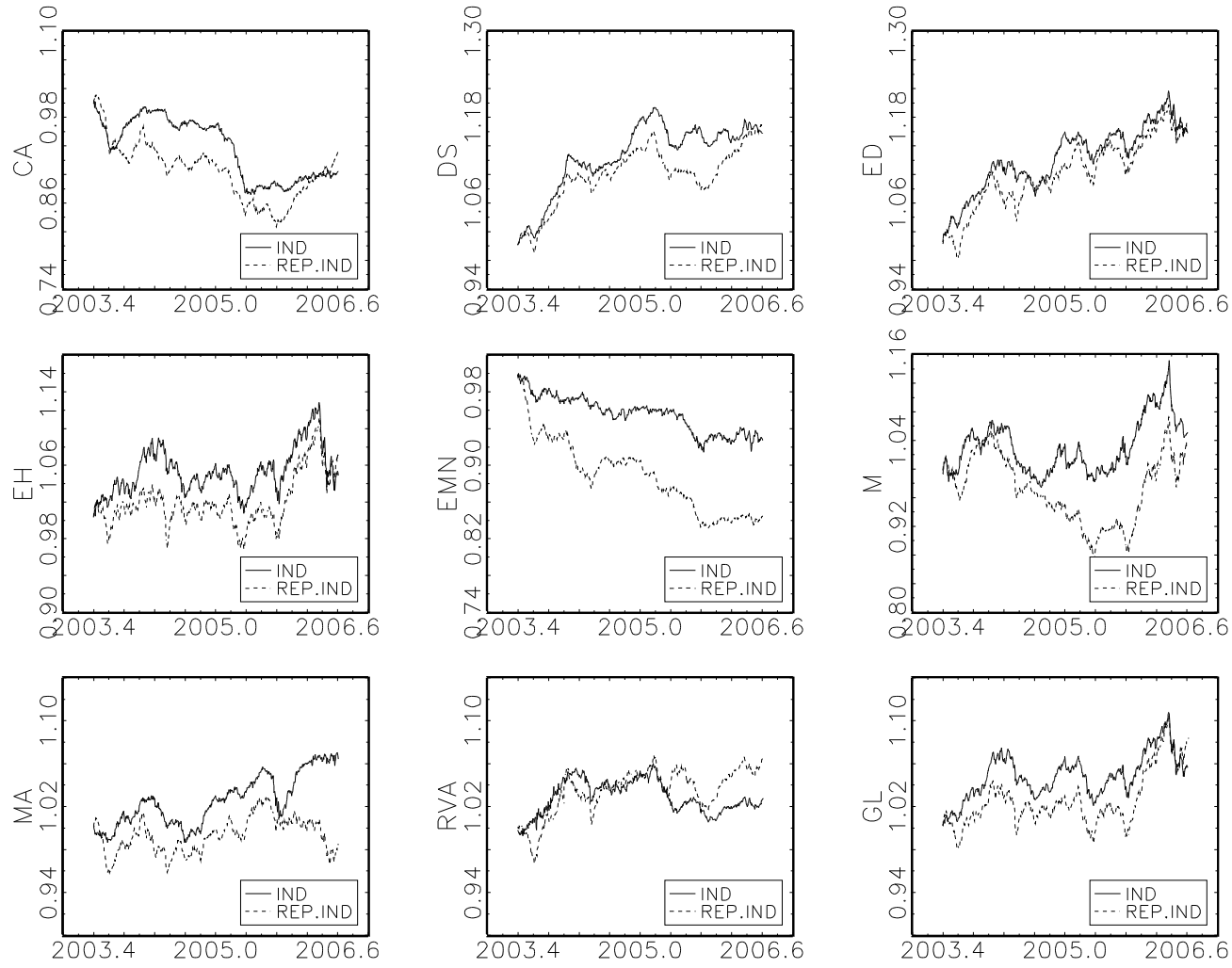

Figure 4: Replicated hedge fund returns versus real hedge fund returns

Finally, also the EH and GL strategies seem to do much worse in the most recent period. MA managers, on the other hand, are very successful in timing the market most recently. They generate returns that are much larger than the passively replicated returns. A few strategies turn out to be less successful, since we can easily replicate the actual returns. This is true for the strategies ED and RVA (and to a lesser extent for DS in the beginning of the sample). The latter is most probably due to the fact that their global risk exposure is dominated by a limited number of risk factors and whereby these exposures only change marginally over time. Table 3 shows that this is

\footnotetext{
${ }^{9}$ Both sides of the CA strategy (long in the bond and short the equity) on General Motors generated huge losses. Huge levering aggravated the situation. A downgrading to junk bonds pushed GM bond prices down, whereas stock prices spiked due to Kirk Kerkonian's offer to raise his stake in GM to about $9 \%$. Both evolutions were opposite to what hedge fund managers thought would happen.
} 
a plausible explanation for the strategies ED, RVA and DS, all dominated by Hyb. Hence passively replicating these exposures is a cost effective alternative.

\section{Conclusion}

In this paper we analyze the underlying dynamics of hedge fund strategies. Given that hedge fund managers often rebalance their portfolio to profit from new trading opportunities, assuming a set of fixed risk exposures through time to measure overall risk is probably not optimal. Analyzing the time-variation in the risk exposures is the main focus of the current paper.

To introduce time-varying parameters, we employ a fixed window rolling-over regression of a multifactor model. This fixed-window then corresponds to the buyand-hold horizon of the hedge fund manager. We find a considerable amount of timevariation in the risk exposures of the hedge fund strategies. Moreover, the rolling-over regressions also show that a number of risk exposures remain hidden in the full sample (long-horizon) regressions. Particular risk factors turn out to be insignificant (significant) when estimated for the full sample of results, while the rolling-over regression indicate that there is, on the contrary, significant (insignificant) exposure to these factors. To get a clear picture of the true risk exposures of the hedge fund returns it is, therefore, important to allow for time-variability.

In a second step, we analyze whether the dynamic strategies translate into excess returns. To this end, we replicate the hedge fund series. Our analysis shows that most of the strategies produce superior returns compared to the replicated series. This suggests that these hedge fund managers are able to generate alpha returns by timing the alternative beta exposures. As Asness (2004a) already indicated, hedge fund risk becomes more and more alternative beta risk and is most likely to add value through this channel in the future. Hence hedge fund managers can still outperform the market, but this is largely driven by a strategy of successful market timing. The latter should come as no surprise. Although it is commonly accepted that timing equity risk and interest rate risk is, at best, difficult, it might well be that this is not the case for other risk factors. Both credit and volatility risk might highlight this point. However, with the continuous advances in finance whereby the market is able to isolate these risk factors and price them individually and hence more efficiently, the timing of these risk factors may become more difficult in the future. The recent explosion we 
have witnessed in the credit derivatives market will ultimately lead to more efficiency in pricing credit risk.

Another important issue is the possible implication of our results when adding hedge funds to a more global portfolio. The often-cited benefits of including hedge funds are their positive effect on overall portfolio risk through increased diversification as well as their role as performance enhancers. However, as is highlighted in our analysis, allowing for time-variation is crucial for a proper risk assessment of any hedge fund strategy. Hence the difficulty in measuring the real underlying correlation benefit and the potential pitfall of using average exposures and correlations. Secondly, if indeed most hedge funds can be defined as (alternative) beta grazers as opposed to alpha hunters, it follows that the inclusion of hedge funds within an overall portfolio becomes more cumbersome as the overall strategic and tactical allocation will differ from the desired allocation. 


\section{References}

[1] Alexander, C. and A. Dimitriu (2005). Detecting Switching Strategies in Equity Hedge Funds, Journal of Alternative Investments 8, 7-13.

[2] Amenc, N., S. Curtis and L. Martellini (2004). The Alpha and Omega of Hedge Fund Performance Measurement. Edhec Risk and Asset Management Research Centre, mimeo.

[3] Asness, C. (2004a). An Alternative Future, Journal of Portfolio Management, Special Issue $30^{\text {th }}$ Anniversary, 94-103.

[4] Asness, C. (2004b). An Alternative Future: Part II, Journal of Portfolio Management 30, 8-23.

[5] Asness, C., R. Krail and J. Liew (2001). Do Hedge Funds Hedge? Journal of Portfolio Management 28, 6-19.

[6] Beckers, S. and J. Smedts (2004). Hedge Funds: Where is the (H)Edge? Tijdschrift voor Economie en Management 49, 847-878.

[7] Boyson, N.M., C.W. Stahel and R. Stulz (2006). Is There Hedge Fund Contagion? NBER Working Paper no. 12090.

[8] Edwards, F.R. and M.O. Caglayan (2001). Hedge Fund Performance and Manager Skill. Journal of Futures Markets 21, 1003-1028.

[9] Fung, W. and D. Hsieh (2004). Hedge Fund Benchmarks: A Risk based Approach. Financial Analyst Journal 60, 65-80.

[10] Fung, W. and D. Hsieh (2002). Asset-Based Style Factors for Hedge Funds. Financial Analyst Journal 58, 16-27.

[11] Géhin, W. and M. Vaissié (2005). The Right Place for Alternative Betas in Hedge Fund Performance: An Answer to the Capacity Effect Fantasy, Edhec Risk and Asset Management Research Centre, mimeo.

[12] Getmansky, M., A.W. Lo and I. Makarov (2004). An Econometric Model of Serial Correlation and Illiquidity in Hedge Fund Returns, Journal of Financial Economics 74, 529-609.

[13] Huber, C. and H. Kaiser (2004). Hedge Fund Factors with Option-Like Structures: Examples and Explanations, Journal of Wealth Management 7, 4960 . 
[14] Ineichen, A. (2002). Absolute Returns: Risks and Opportunities of Hedge Fund Investing, John Wiley \& Sons Inc. Hoboken New Jersey, pp. 514.

[15] Loeys, J. and L. Fransolet (2004). Have Hedge Funds Eroded Market Opportunities? JPMorgan, October.

[16] Jaeger, L. and C. Wagner (2005). Factor Modelling and Benchmarking of Hedge Funds: Can Passive Investments in Hedge Funds Deliver?, Journal of Alternative Investments 8, 9-36.

[17] Liang, B. (1999). On the Performance of Hedge Funds, Financial Analysts Journal 55, 72-83.

[18] Malkiel, B.G. and S. Saha (2005). Hedge Funds: Risk and Return, Financial Analysts Journal, 61, 80-88.

[19] McGuire, P., E. Remolona and K. Tsatsaronis (2005). Time-Varying Exposures and Leverage in Hedge Funds. BIS Quartely Review March, 59-72.

[20] Ranaldo, A. and L. Favre (2003). How to Price hedge Funds: From Two- to Four-Moment CAPM, Edhec Risk and Asset Management Research Centre, mimeo.

[21] Sharpe, W. (1992). Asset Allocation Management Style and Performance Measurement. Journal of Portfolio Management 18, 7-19. 
Appendix A
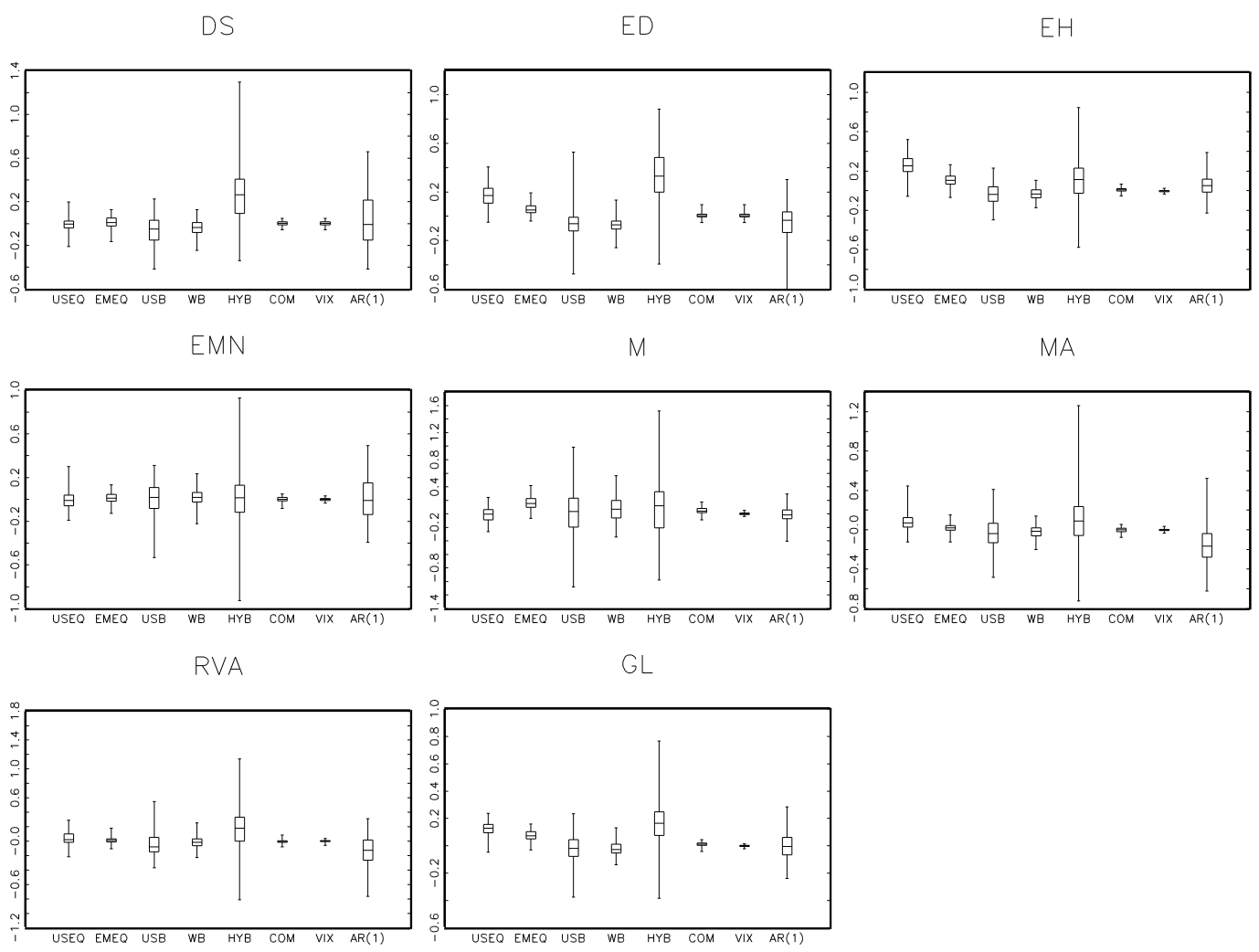

Figure 5: Distribution of the rolling-over risk exposures 